\title{
A silicon analyser for the OSIRIS spectrometer: An analytical and Monte Carlo simulation study
}

\author{
A. Perrichon, F. Fernandez-Alonso, M. Wolff, \\ M. Karlsson, F. Demmel
}

\section{Published version information}

Citation: A Perrichon et al. "A silicon analyser for the OSIRIS spectrometer: an analytical and Monte Carlo simulation study." Nuclear Instruments and Methods in Physics Research Section A, vol. 947 (2019): 162740.

DOI: $\underline{\text { 10.1016/i.nima.2019.162740 }}$

C2019. This manuscript version is made available under the CC-BY-NC-ND 4.0 Licence.

This version is made available in accordance with publisher policies. Please cite only the published version using the reference above. This is the citation assigned by the publisher at the time of issuing the AAM. Please check the publisher's website for any updates. 


\title{
A silicon analyser for the OSIRIS spectrometer: An analytical and Monte Carlo simulation study
}

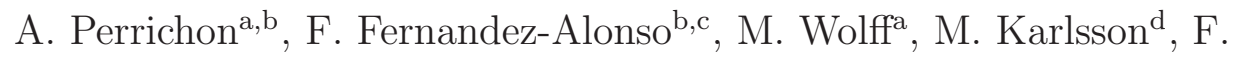 \\ Demmel $^{\mathrm{b}, *}$ \\ ${ }^{a}$ Department of Physics and Astronomy, Uppsala University, 75237 Uppsala, Sweden \\ ${ }^{b}$ ISIS Facility, Rutherford Appleton Laboratory, Chilton, Didcot, Oxfordshire OX11 0QX, \\ United Kingdom \\ ${ }^{c}$ Department of Physics and Astronomy, University College London, Gower Street, \\ London WC1E 6BT, United Kingdom \\ ${ }^{d}$ Department of Chemistry and Chemical Engineering, Chalmers University of \\ Technology, 41296 Göteborg, Sweden
}

\begin{abstract}
The indirect time-of-flight near-backscattering spectrometer OSIRIS at the ISIS Facility is being upgraded with the addition of a silicon analyser. The new analyser bank will allow to increase the dynamic range, giving access to relaxation times up to $400 \mathrm{ps}$, and provide a further dimension in momentum transfer detection with the help of position-sensitive detectors. Here we present analytical calculations for the energy resolution and an extensive Monte Carlo simulation study to asses the performance of the new analyser bank. Simulation and calculation agree perfectly and confirm the initial design parameters of the spectrometer. The simulations predict similar detected intensity but with a higher resolution compared to the existing analyser setup using pyrolytic graphite crystals. Furthermore, the simulations stress the importance of the sample height for this setup and point toward a necessary further upgrade of the guide system. Further improvements of the energy resolution might be achieved with a pulse shaping chopper.
\end{abstract}

Keywords: neutron spectrometer, Monte Carlo simulations

\footnotetext{
*Corresponding author

Email address: franz.demmel@stfc.ac.uk (F. Demmel)
} 


\section{Introduction}

The backscattering technique with neutrons was developed about five decades ago in Garching, Germany [1, 2]. This type of instrument promised the highest energy resolution of about $1 \mu \mathrm{eV}$ at that time and hence access to ns relaxation times. Most of the following developments focused on enhancing the flux and, nowadays, the most modern instruments also exploit phase space transformation $[3,4,5,6]$.

At pulsed sources, backscattering from an analyser crystal has been implemented in combination with time-of-flight (TOF) to analyse the energy of the incident neutrons. One of the first instruments of this type was IRIS at the ISIS Facility (Rutherford Appleton Laboratory, Didcot, UK) [7, 8]. The instrument is different from classical backscattering spectrometers in the sense that it uses cooled pyrolytic graphite analysers in a near-backscattering geometry. This results in a relaxed energy resolution of about $15 \mu \mathrm{eV}$, but with a significantly increased dynamic range. The combination of high resolution and wide range in momentum transfers $(Q)$ with a large dynamic range allowed novel applications in cold neutron spectroscopy. Based on a similar design as IRIS, the OSIRIS spectrometer at the ISIS Facility achieved a large gain in intensity by using a supermirror guide and an increased analyser unit $[9,10]$. At the other end an improvement of the energy resolution towards the $\mu \mathrm{eV}$ regime at a spallation source was achieved on BASIS (Oak Ridge National Laboratory, TN, USA), by combining a long flight path with a silicon analyser [11]. Following this concept a series of TOF backscattering spectrometers have been built or proposed $[12,13,14,15,16]$. At reactor sources the approach combining a crystal analyser with a time-of-flight primary spectrometer has been proposed and implemented as well [17, 18, 19, 20].

The cold TOF spectrometer OSIRIS has been described in detail in references $[9,10,21,22]$ and hence we will only provide a short overview. OSIRIS has been recently upgraded with a moveable Beryllium filter to remove the second order reflection of the pyrolytic graphite analyser [23], and its energy resolution has been modelled with Monte Carlo simulations [24]. It is mainly used to study low-energy excitations, e.g. the magnetic field dependence of the spin resonance in $\mathrm{CeCoIn}_{5}$ [25], or spinon confinement in $\mathrm{Yb}_{2} \mathrm{Pt}_{2} \mathrm{~Pb}$ [26], and for quasielastic neutron scattering experiments probing stochastic dynamics, e.g. for catalysis $[27,28]$, or the mobility of ions in battery materials [29].

To further improve the performance of the instrument with respect to 
energy resolution, and allow novel challenging studies of, for instance, ionic diffusion of weakly incoherently scattering ions in battery materials, an upgrade was proposed to implement a $\mathrm{Si}(111)$ analyser unit allowing energy resolution down to $\Delta E \approx 10 \mu \mathrm{eV}$ [30]. This silicon analyser (SA) will be constituted of two banks of curved analysers, positioned on the vacant side inside the vacuum tank of the secondary spectrometer. Each analyser unit will focus the neutrons onto an image point above or below the sample position. Position-sensitive ${ }^{3} \mathrm{He}$ detectors (PSD), positioned above and below the horizontal, sample scattering plane $\left(Q_{x}, Q_{y}\right)$, will be used to register the Bragg-reflected neutrons. The use of PSD's allows to access the vertical direction in momentum transfer $\left(Q_{z}\right)$ for single-crystal studies and gives the opportunity to correct for the small path-length differences in the secondary spectrometer. More details on the engineering design can be found in reference 30 .

Here we present analytical calculations of the energy resolution and Monte Carlo simulations using the McStas programme package to assess the performance of the new SA setup.

\section{Analytical considerations}

The OSIRIS instrument has been described in detail previously $[9,10$, $22,21]$ and hence we will only provide a brief account of parts relevant for this work. The OSIRIS instrument views the $25 \mathrm{~K}$ cold hydrogen moderator at the Target Station 1 of the ISIS Neutron and Muon Source. A curved supermirror guide $(m=2)$ transports the neutrons to the sample position $34.00 \mathrm{~m}$ away from the moderator. At the end of the guide a $1.5 \mathrm{~m}$ long focusing guide section $(m=3.6)$ reduces the beam cross section from $43 \times$ $65 \mathrm{~mm}$ to $22 \times 44 \mathrm{~mm}$. The secondary spectrometer consists of a large analyser bank and 42 half inch diameter ${ }^{3} \mathrm{He}$ detectors with an active length of $38 \mathrm{~mm}$. The neutrons are scattered in a near-backscattering geometry from more than $8000 \times 1 \mathrm{~cm}^{2}$ sized pyrolytic graphite crystals into the detectors. Two reflections from the graphite crystals can be used: the (002) and (004) reflections define final energies of $1.845 \mathrm{meV}$ and $7.38 \mathrm{meV}$, respectively. The analyser bank is cooled to $10 \mathrm{~K}$ to reduce thermal diffuse scattering.

In a previous study it was shown that the current energy resolution is limited by the geometry of the analyser unit [24]. To further improve the energy resolution, silicon is considered as analyser material, which is the standard at classic backscattering spectrometers, and nowadays also on high-resolution 
TOF backscattering instruments [31]. There are several advantages in using silicon compared to the present pyrolytic graphite. Since $\mathrm{Si}(111)$ has no second order reflection, there is no need for the installation of a cooled beryllium filter. Furthermore, silicon has extremely low thermal diffuse scattering, as it was recently demonstrated by the DNA spectrometer (J-PARC, Naka-gun Ibaraki, Japan) that achieved a very high signal to noise ratio approaching $10^{5}[12,13,14]$. However, there is a disadvantage with silicon crystals concerning its neutron reflectivity: the crystals are too perfect for the required resolution, in particular, for the more relaxed resolution in near-backscattering geometry. For the $\mathrm{Si}(111)$ reflection, the energy resolution contribution from the Darwin width is $0.077 \mu \mathrm{eV}$ [31] and, hence, about a factor 100 too small for OSIRIS. That mismatch with the resolution of the primary spectrometer would result in a substantial intensity loss. To overcome this limitation, standard practice involves the elastic deformation of the crystals, which increases the reflected intensity. This results in a gradient in lattice spacing, $d$, which depends on the curvature radius of the analysers, $R_{A}$, and the thickness of the wafers, $D$, according to Eq. (1) [3], with $\mu_{\text {eff }}$ being an average Poisson ratio, and the small contribution from the Darwin width being omitted.

$$
\frac{\Delta d}{d}=\mu_{e f f} \frac{D}{R_{A}}
$$

Another advantage of the new setup is that position-sensitive detectors will be used. The existing graphite analyser on OSIRIS focuses all reflected neutrons to the detector position and hence a position-sensitive detector is not useful. On the other hand, the chosen elliptical geometry with a focus point at the detector position has no path-length differences in the secondary spectrometer. However, the SA will focus the neutrons on an image point below or above the sample position and therefore a linear PSD can analyse $Q_{z}$ and therefore correct for final flight-path differences.

To calculate the expected energy resolution for the SA setup, we are using the same formalism as previously published [16, 24]:

$$
\frac{\Delta E}{E}=2\left\{\left(\frac{\Delta t}{t}\right)^{2}+\left(\frac{\Delta L}{L}\right)^{2}+\left(\frac{\Delta d}{d}\right)^{2}+(\cot \theta \Delta \theta)^{2}\right\}^{1 / 2}
$$

For monochromatic focusing conditions of a Bragg-reflecting spherically-bent crystal, the focusing distance $f$ is $f=\frac{R_{A}}{2} \sin \theta$ with $\theta$ the Bragg angle, and the object and image distances are equal to $2 f$ [32]. 
The SA will sit inside the existing vacuum tank at a distance of $880 \mathrm{~mm}$ from the sample position. The curvature radius shall be $880 \mathrm{~mm}$ and the radius centre point shall be $110 \mathrm{~mm}$ below or above the sample. The angular term of the analyser resolution is determined by the near-backscattering Bragg angle $\theta=83^{\circ}$. The Bragg angle is strongly interrelated with the resolution and directly linked to the shift of the focus point. On the other side the Bragg angle has to be balanced with the solid angle opening towards the analyser bank, which determines the intensity. The vertical opening angle towards the analysers from the point of view of the sample position is limited by the positioning of the detectors on a radius of $240 \mathrm{~mm}$ around the sample position. The smaller the shift of the focus point, the nearer the detectors will be positioned on the sample plane and, hence, reduce the solid angle available to the analyser crystals, because of shadowing of the scattered neutrons. The $110 \mathrm{~mm}$ shift of the radius point was found to be a good compromise between achievable resolution and available solid angle. The divergences are defined through the sample size (assumed to be $10 \mathrm{~mm}$ high) and the pixel size of the linear PSD's which will be around $15 \mathrm{~mm}$. This sample size is about the minimum size which is normally applied to single-crystal experiments up to now. More details of the influence of sample size on energy resolution will be presented in the section devoted to the simulations. For a Bragg crystal with incoming beam divergence $\alpha_{i}=1.3^{\circ}$ and outgoing divergence $\alpha_{f}=0.9^{\circ}$ and negligible mosaic spread, $\Delta \theta$ can be written in the following form [33].

$$
\Delta \theta=\frac{\alpha_{i} \alpha_{f}}{\sqrt{\alpha_{i}^{2}+\alpha_{f}^{2}}}
$$

For the contribution of the elastic bending of the wafers, we assume a wafer thickness of $D=0.8 \mathrm{~mm}$, which will be the thickness to be used in the construction. With all these input parameters, we calculate the energy resolution around the final energy $E_{f}=2.11 \mathrm{meV}$.

For the time-of-flight uncertainties $\Delta t$ the main contribution stems from the moderator pulse, which is about $125 \mu \mathrm{s}$ at $\lambda=6 \AA$. Interpolated moderator widths determined from diffraction experiments have been used for different wavelengths [24]. Another term is the spread from the guide $\Delta t_{\text {guide }}$. The latter can be estimated by taking into account path-length differences in the guide $(m=2)$ with an opening of $43 \mathrm{~mm}$. By calculating the maximum number of reflections over $32 \mathrm{~m}$, about 10, we can estimate the length and therefore the time spread of the neutrons due to different paths. We get a 
maximum delay of $\Delta t=22 \mu \mathrm{s}$ for $6 \AA$ neutrons, from which we deduce a contribution of $\Delta t_{\text {guide }}=11 \mu$ s to the spread in time. For the sample size TOF contribution we assume a diameter of $10 \mathrm{~mm}$ for the sample which can be transferred into a time spread of $8 \mu \mathrm{s}$. On the secondary spectrometer, we assume no further time spread from different path lengths due to the use of PSD's and only a $\Delta t_{\text {detector }}=7 \mu$ s spread from the ${ }^{3}$ He detectors. All timeof-flight contributions are added quadratically assuming they are statistically independent.

Figure 1 shows the resulting energy resolution for energy transfers around the elastic line. The calculated resolution for the PG analyser agrees perfectly with the measured value from a vanadium standard at the elastic line and with Monte Carlo simulations [24]. With the SA, the energy resolution will be $11 \mu \mathrm{eV}$ at the elastic line, hence an improvement by about a factor 2.5 . Included is also the contribution solely from the primary spectrometer, which now dominates the resolution, in contrast to the PG(002) case, where the secondary spectrometer is the major contribution to the energy resolution [24]. The calculated contribution of the secondary spectrometer is $\Delta E_{f}=$ $7.2 \mu \mathrm{eV}$ at the elastic line.

With the SA, in the time domain, relaxation times up to around 400 ps will be accessible and in total about 3 orders in magnitude in relaxation times can be covered over a wide range of wave vectors $\left(Q=0.2-2.0 \AA^{-1}\right)$ in a single setting. This increases the versatility of OSIRIS that will then operate synchronously the PG analyser, the SA, and the diffraction bank.

\section{Simulations}

The simulations were performed with the McStas ray-tracing package $[34,35,36]$. As source we used the ViewModISISver1 component, which links to a file characterising the $25 \mathrm{~K}$ cold hydrogen moderator leading to the OSIRIS and IRIS instruments.

For the transport of neutrons, the guide and the guide_curved components with the standard reflectivity profile were used, with $\alpha=6.07$ the linear decrease in reflectivity beyond $m=1$. The curvature radius of the guide is $R=2050 \mathrm{~m}$ and corresponds to a characteristic wavelength of $\lambda \approx 2 \pi \sqrt{(2 a / R)} / k_{\perp}=1.92 \AA$ with $k_{\perp}=0.0214 \AA^{-1}$ the maximum wavevector for a $m=2$ guide and a guide width of $a=43 \mathrm{~mm}$. At this characteristic wavelength, the transmission of the curved guide is reduced to $2 / 3$ compared 


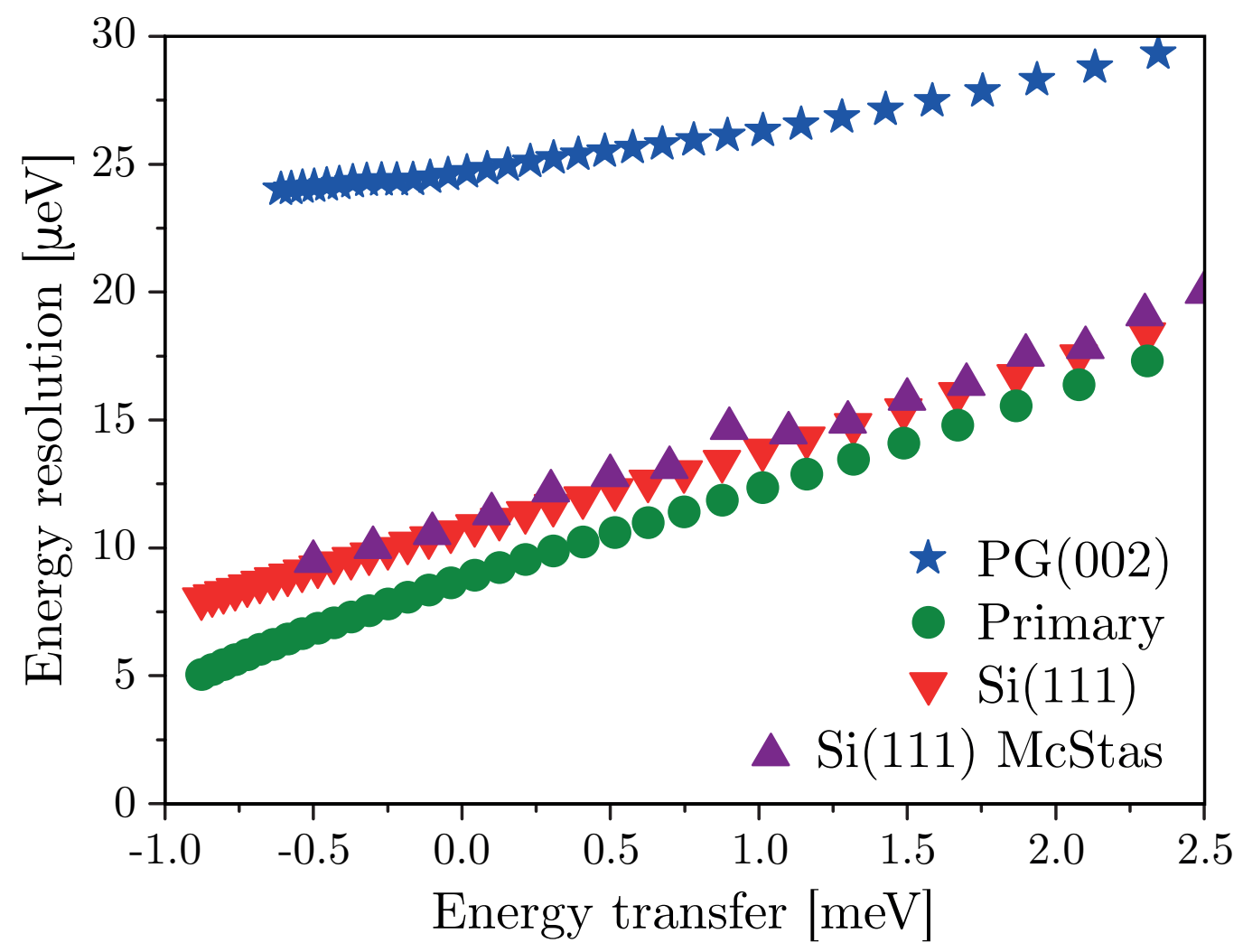

Figure 1: Analytically calculated total energy resolution of the SA (inverted triangles) and pyrolytic graphite analyser $\mathrm{PG}(002)$ (stars). In addition, the contribution from the primary spectrometer alone is shown (circles). The energy resolution of the SA calculated from the McStas simulations is also shown for comparison (triangles). 

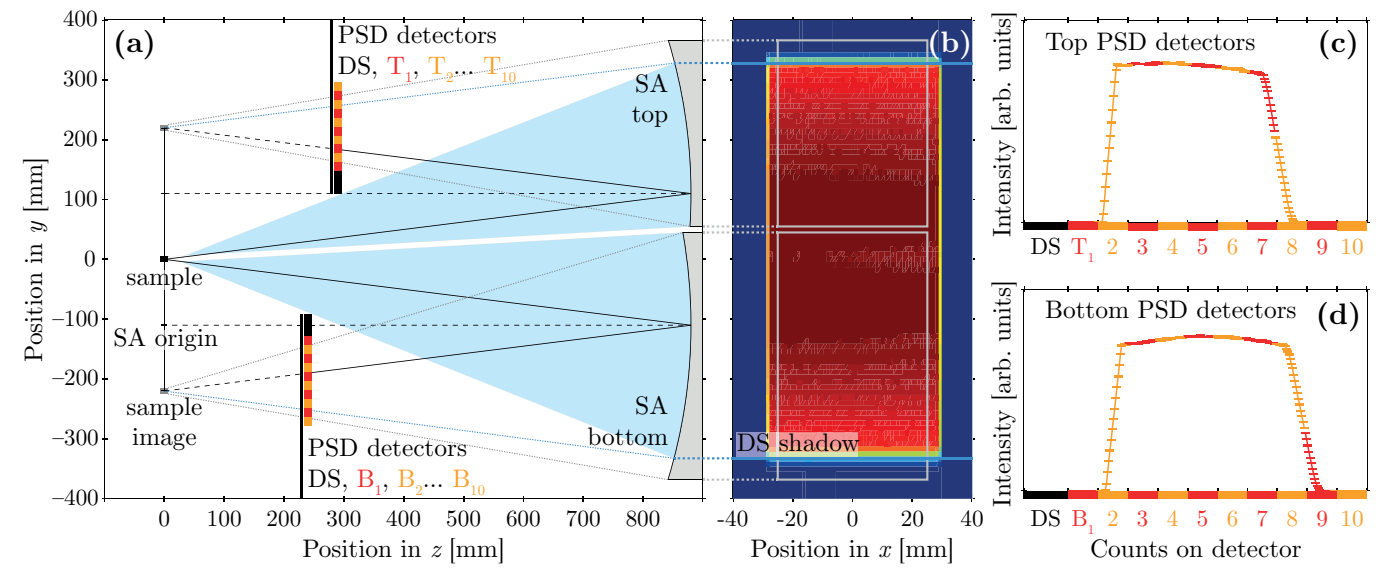

Figure 2: (a) Scheme of the silicon side of the secondary spectrometer modeled in McStas. (b) Neutron counts on the analyser (2D PSD monitor component). Dimension and position of the SA top and bottom part are represented by grey lines, and detector dead-spot (DS) shadow positions by blue lines. Pixel-wise neutron counts on the PSD top (c) and bottom (d) detectors.

to a straight guide and indicates a lower limit of useful wavelengths. Incoming energies up to about $20 \mathrm{meV}$ and hence energy transfers up to $18 \mathrm{meV}$ can be exploited on OSIRIS, as exemplified on IRIS [37, 38]. At the end of the guide a $m=3.6$ straight focusing section reduces the beam area from $43 \times 65 \mathrm{~mm}^{2}$ to $22 \times 44 \mathrm{~mm}^{2}$. The guide ends $250 \mathrm{~mm}$ before the sample position.

The incoherent and the tunneling_sample components from McStas are used as samples, which both emulate an isotropic incoherent scatterer either for elastic or inelastic scattering. The sample geometry is defined by a hollow cylinder, with thickness of $2 \mathrm{~mm}$ and an outer diameter of $12 \mathrm{~mm}$. The sample height is set as a variable parameter, with a default value of $10 \mathrm{~mm}$.

The SA is modeled using two instances of the PerfectCrystal component, representing the top and bottom part of the analyser, respectively, as illustrated in Fig. 2(a). Note that the PerfectCrystal component describes a curved crystal on a sphere. The position of the SA is fixed to a distance of $880 \mathrm{~mm}$ from the sample. The curvature radius is then defined as $R_{A}=880+\Delta R_{A} \mathrm{~mm}$, with $\Delta R_{A}$ being the offset leading to under or over-focusing of the sample image.

The height of the SA is set to $413 \mathrm{~mm}(-368 \leq y \leq 45 \mathrm{~mm}$ with the sample at the origin) for the bottom part and $311 \mathrm{~mm}(55 \leq y \leq 366 \mathrm{~mm})$ 
for the top part, which represent an angular coverage of $40-45^{\circ}$ in $\phi$ (sampleheight dependent, blue area in Fig. 2(a)). The analyser width is set to $50 \mathrm{~mm}$, which corresponds to the width of one detector tube at the detector position, and represents $3.25^{\circ}$ of the $142.5^{\circ}$ angular coverage in $\theta$. We only consider one scattering angle in the horizontal plane assuming no significant change of resolution and performance with $2 \theta$. The reflection condition of the analyser is determined by the Bragg law considering scattering angles close to $83^{\circ}$ and the $d$-spacing of $\operatorname{Si}(111)$ lattice planes, $d_{111}=3.135 \AA$. The spread in $d$-spacing is determined from Eq. (1), with values close to $\Delta d / d=3 \cdot 10^{-4}$.

The top and bottom detectors are modeled by several superimposed monitor components, including the PSD_monitor and the TOF2E_monitor components, measuring the $2 \mathrm{D}$ spatial distribution and the final energy of neutrons converted from the TOF at the detector position, respectively. Linear position-sensitive ${ }^{3} \mathrm{He}$ tubes, with $1 / 2$ inch diameter, will be used as detectors. They exhibit a dead end of about $37 \mathrm{~mm}$, which will interfere with the scattered neutrons and has to be taken into account in the simulations. It is not relevant for the resolution considerations, however, the solid angle and therefore the intensity is directly influenced by the detector dead end. The position sensitive aspect of the detector is modeled by dividing the detector height in 10 sections, represented by a stack of 10 monitor components with a pixel height of $15 \mathrm{~mm}$ (labeled $\mathrm{B}_{1}$ to $\mathrm{B}_{10}$ and $\mathrm{T}_{1}$ to $\mathrm{T}_{10}$ ) and a width of $12.7 \mathrm{~mm}$. The monitor arrays are positioned on a $240 \mathrm{~mm}$ and $290 \mathrm{~mm}$ radius from the sample for the bottom and top part, respectively. The larger radius for the top detector array results from engineering constraints around the sample bin of the spectrometer. The detector dead-spot (DS) is simply represented, at the detector position, by an offset of the monitor arrays. The shadow caused by the dead-spot (DS) on the silicon analyser (Fig. 2(b)) is treated by adding a neutron-absorbing mask at the position of the detector, modeled beforehand by a Beamstop component. The neutron counts on each pixel of the top and bottom PSD detectors are shown in Fig. 2(c) and 2(d). The size of the sample image at the position of the detector depends on the sample height; for a $3 \mathrm{~cm}$ tall sample, the entire analyser and detector array is used.

The line shape parameter full width at half maximum (FWHM), which represents the energy resolution and can be directly compared to the analytical calculations, is extracted from the simulated spectra through a fit with a Gaussian line shape. The curve is fitted to the highly asymmetric simulation curves in a restricted energy range around the peak value. 

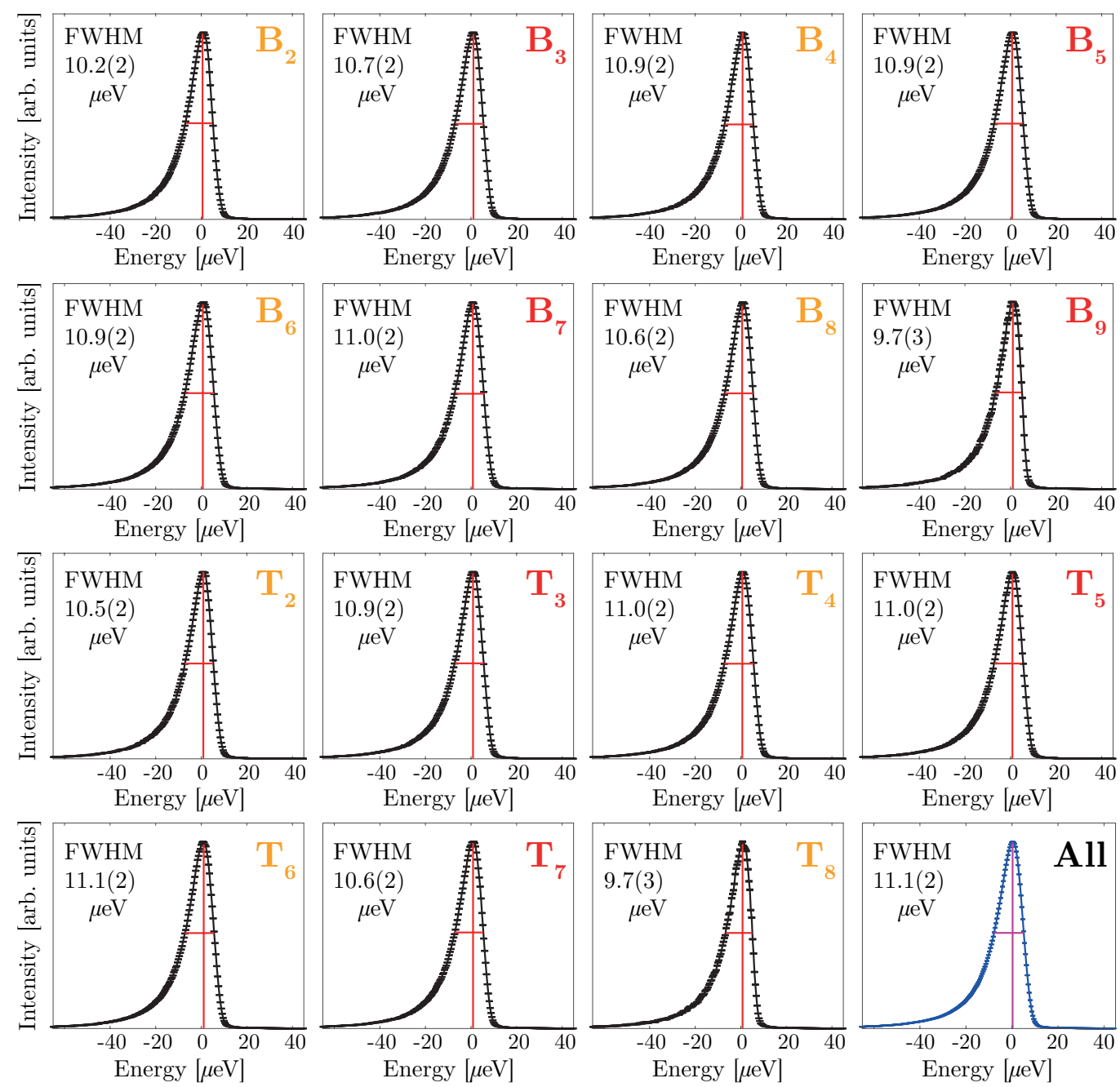

Figure 3: Line shape, calculated from the time-of-flights, zero-shifted, measured on each pixel constituting the monitor arrays associated to the top part $\left(\mathrm{T}_{2}\right.$ to $\left.\mathrm{T}_{8}\right)$ and bottom part $\left(\mathrm{B}_{2}\right.$ to $\left.\mathrm{B}_{9}\right)$ of the SA, together with their FWHM. The sum of the 15 individual pixel line shapes is represented in blue (labeled "All") and has a FWHM of 11.1(2) $\mu \mathrm{eV}$. 


\section{Results and Discussion}

In the following section we will consider the influence of different parts of the instrument to the resolution before we discuss the full results.

\section{Pixel-wise and overall resolution}

Fig. 3 shows the line shape, calculated from the time-of-flights, measured on each pixel constituting the monitor arrays associated to the top and bottom part of the SA. The line shapes are zero-shifted, i.e. the energy corresponding to the maximum of the line shape is shifted to zero. Note that only the pixel with neutron counts are represented ( $c f$. Fig. 2(c), 2(d)). For a specific pixel, the FWHM of the line shape is estimated, from a Gaussian fit, in the range 9.7-11.1 $\mu \mathrm{eV}$. The slightly better resolution of the detector pixels with lower neutron counts $\left(\mathrm{B}_{2}, \mathrm{~B}_{9}\right.$, and $\left.\mathrm{T}_{2}, \mathrm{~T}_{8}\right)$ is explained by their partial exposure to the sample images, which makes the effective sample height smaller, thus reducing its contribution to the resolution. We also report in Fig. 3 the sum of the individual pixels. The overall total energy resolution is about $11.1(2) \mu \mathrm{eV}$ in excellent agreement with the analytical calculations.

In order to isolate the contribution of the SA from the primary spectrometer, we added a DiskChopper component directly after the source component, to reduce the time-of-flight spread from $c a 125 \mu$ s to $c a 12 \mu \mathrm{s}$. Note that the average line shape is now symmetric (Fig. 4), which confirms that the asymmetry of the total time-of-flight (primary and secondary) originates from the moderator asymmetric pulse. Note that an overall signal to background ratio of more than four orders of magnitude should be possible in such a configuration, a value which has already been demonstrated on the DNA spectrometer. The FWHM of the line shape is $6.0(1) \mu \mathrm{eV}$, which then corresponds mostly to the secondary spectrometer contribution of the total resolution. Adding quadratically this contribution from the secondary spectrometer with the contribution from the primary spectrometer, which was estimated to be $8.5 \mu \mathrm{eV}$ [7], results in a total resolution of $10.4 \mu \mathrm{eV}$ in good agreement with the simulation.

The width of $6.0(1) \mu \mathrm{eV}$ is then a good prediction for an OSIRIS spectrometer equipped with a pulse shaping chopper. The disadvantage of a pulse shaping chopper option is the drastically reduced dynamic range, reasoned now by the width of the moderator pulse and the distance between chopper and moderator [16]. The dynamic range can be increased by a multi-slit 


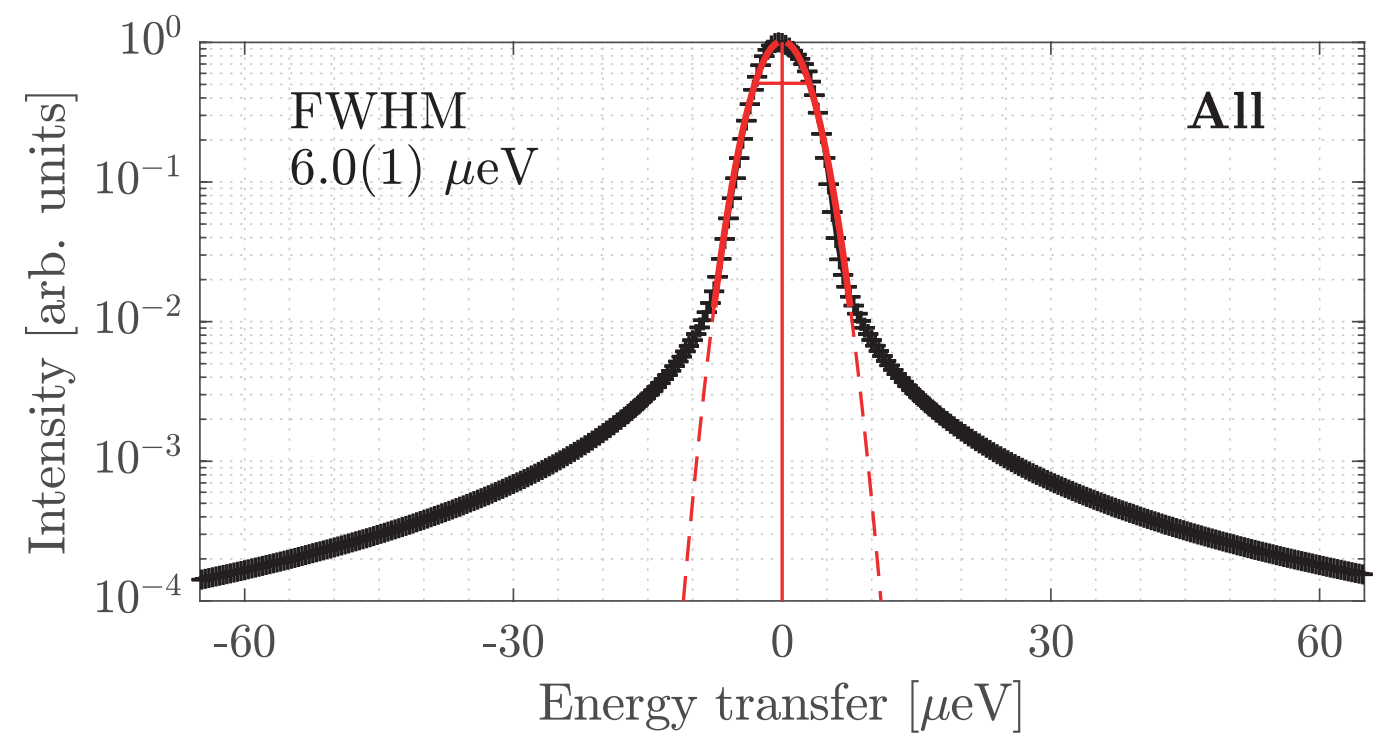

Figure 4: Simulated line shape on a logarithmic scale, calculated from the sum of the zero-shifted lines over all pixel detectors. The incoming pulse was chopped to a length of $12 \mu s$. As a dashed line, a Gaussian fit is included to determine the FWHM.

option for the choppers. In combination with phase changes at the chopper system a wide dynamic range can be recovered. That option has been implemented in the DNA spectrometer [12]. The shortening of the pulses and the sequential measuring have a direct impact on the counting rate. With the chosen Bragg angle it would not be possible to achieve an even better energy resolution, for example, comparable to the DNA spectrometer [12]. The small size of the secondary spectrometer limits the possibilities towards an even higher resolution. To achieve an improvement of the energy resolution by a factor 2 with all the implications on operation and intensity of the spectrometer this option seems to be not very favorably. A complete new design would be necessary to move towards $1 \mu \mathrm{eV}$ resolution, which was already proposed some time ago [16].

\section{Resolution dependence in relation to SA geometry}

Figure 5 shows the dependence of the intensity (a) and FWHM (b) of the line shape with the vertical distance (offset) of the focus point from the sample ( $c f$. Fig. 2(a)). That offset is directly correlated to the Bragg angle at the SA and therefore to the resolution. With increasing offset also the path length differences play a larger role and hence the resolution worsens with 
a single detector compared to the pixelated detection mode. The detector dead-spot creates a shadow on the SA and consequently the detected intensity decreases, depending on the offset value. On the other side, the offset value is directly linked to the Bragg angle and hence the resolution. At an offset value of $120 \mathrm{~mm}$ the whole $\mathrm{SA}$ is exposed to the neutrons. We have chosen a distance of $110 \mathrm{~mm}$ (box in Fig. 5) as the most sensible compromise between resolution and solid angle.

Figure 5(c) shows the dependence of the FWHM with the $\Delta R_{A}$ parameter, which represents a modulation of the curvature radius of the SA around its value of $880 \mathrm{~mm}$ when keeping the distance between sample axis and analyser constant at $880 \mathrm{~mm}$, i.e. having the SA in over-focusing $\left(\Delta R_{A}<0\right)$ or in under-focusing conditions $\left(\Delta R_{A}>0\right)$. The energy resolution shows a broad minimum for values of $\Delta R_{A}$ in the range of 0-40 $\mathrm{mm}$.

\section{Resolution dependence with sample height}

Figure 6 shows the dependence of the intensity and FWHM of the line shape with sample height. As expected, the intensity varies linearly with the sample height. The energy resolution saturates at $c a 11 \mu \mathrm{eV}$ for a small sample $(h=10 \mathrm{~mm})$ and increases linearly to $c a 18 \mu \mathrm{eV}$ for a $30 \mathrm{~mm}$ sample. This dependence opens up the possibility to trade intensity against resolution. With a modest increase of the FWHM by $50 \%$ the measured intensity can be increased by a factor 3 . The resolution of the PG analyser is dominated by the elliptical geometry [24] and, hence, the influence of the sample height is quite small. By eliminating that contribution in the resolution, in Eq. (2), the $\Delta \theta$ term is now dominated by the divergence contribution from the sample height.

For an ideal reflection the sample size defines the size of the picture on the focus point, which is about $240 \mathrm{~mm}$ behind the detectors position. Therefore the vertical Q-direction of a small sample can be resolved on the PSD.

To use the highest resolution without losing too much intensity with a smaller sample an important step would be to focus the neutrons on a smaller sample spot. By using modern guide geometries, like elliptic or parabolic focusing sections, smaller sample spots can be achieved and a gain in intensity of a factor of about 5 on a $1 \mathrm{~cm}^{2}$ sample size are within reach for OSIRIS.

\section{Resolution dependence with energy transfer}

The dependence of the energy resolution with the energy transfer is shown in Fig. 1. The trend calculated from the McStas data is in excellent agreement 
with the analytical calculation as shown in this figure.

\section{Resolution with the Si(333) reflection}

The present OSIRIS supermirror guide delivers neutrons up to about $20 \mathrm{meV}$ with high intensity and beyond this energy the cut-off due to the curvature reduces the intensity smoothly. Hence, it will be possible to use the $\mathrm{Si}(333)$ reflection with a suitable setting of the bandwidth choppers. That instrument setting could deliver valuable information on a much wider $Q$-range up to $Q=6 \AA^{-1}$. A much wider wave vector range is beneficial in studies of local dynamics to properly asses the elastic incoherent structure factor. Also jump diffusion models for translational dynamics rely on a wide-enough $Q$-range. We also simulated the $\mathrm{Si}(333)$ reflection of the $\mathrm{SA}$ by decreasing the $d_{h k l}$ from $3.135 \AA$ to $1.045 \AA$, and increasing the incident neutron energy from $2.0-2.2 \mathrm{meV}$ to $18.0-19.8 \mathrm{meV}$. This increases the maximum $Q$ to $c a 6 \AA^{-1}$, at the cost of an increased energy resolution, simulated as FWHM $=77.8(3) \mu \mathrm{eV}$ at the elastic line (Fig. 7). The final energy of the $\mathrm{Si}(333)$ reflection is a factor 9 larger, however, with a smaller wavelength the pulse width from the moderator decreases and therefore the energy resolution is smaller than the factor 9 increase. In fact, the energy resolution is smaller than the presently possible value for the PG(004) setup, which is $\mathrm{FWHM}=99 \mu \mathrm{eV}$ with still a smaller $Q$-range.

\section{Intensity comparison}

Finally, we discuss the simulated intensities of the PG(002) analyser and the $\operatorname{Si}(111)$ analyser, considering the standard sample height of $10 \mathrm{~mm}$ ( $c f$. Fig. 7). Calculating the integral under the spectral curves we obtain a factor 1.8 higher intensity for the SA compared to the PG analyser, which is due to the increased solid angle. That factor does not include the losses due to the transmission of the Beryllium filter on the PG side, about 60\% [23]. Hence, it can be expected that, considering the same sample size, the SA setup will outperform the PG analyser not only in the view point of resolution but also intensity. Because the analysing energy of $\mathrm{PG}(002)$ and $\mathrm{Si}(111)$ are very similar, both analysers, in addition to the diffraction bank, can be operated concurrently for one sample, which significantly increases the output and versatility of OSIRIS. 


\section{Conclusions}

Monte Carlo simulations and analytical calculations have been performed to describe the energy resolution of a new secondary spectrometer of the OSIRIS instrument. The use of silicon analysers will improve the energy resolution by about a factor 2.5 and relaxation times up to 400 ps will be accessible, compared to about 160 ps previously. The predicted FWHMs of the analytical calculations and the results from the simulations are in excellent agreement. The simulations guided the design to choose the optimum geometric parameters, and evinces a strong link between sample height and resolution in this high resolution setup. As a consequence a new focusing guide would benefit this SA setup. Preliminary Monte Carlo simulations estimate a gain in intensity of a factor 5 at the sample position on a $1 \mathrm{~cm}^{2}$ spot size with an elliptically curved supermirror guide. Due to the larger solid angle, the measured intensity on the SA will be similar to the one obtained on the pyrolytic graphite side. The concurrent use of the PG analyser and the SA will lead to an increase in intensity by about a factor 3 with respect to the graphite analyser alone. The simulations also showed the limitations of this design, which is constrained by the existing design of the secondary spectrometer. Implementation of a pulse shaping chopper would allow to gain another factor 2 in resolution with serious consequences for dynamic range and intensity. However, to go beyond this value, a completely new designed secondary spectrometer is necessary. The OSIRIS silicon analyser is now under construction and envisaged to be ready to the user community in the second half of 2021.

\section{Acknowledgments}

This work was supported by the Science and Technology Facilities Council (STFC) and by the Swedish Research Council (VR, Grant 2016-06958).

\section{References}

[1] B. Alefeld, Rückstreuversuche hoher auflösung mit neutronen, Bayerische Akademie der Wissenschaften 11 (1967) 109. URL http://publikationen.badw.de/en/003384189 
[2] H. Maier-Leibnitz, Grundlagen für die beurteilung von intensitäts- und genauigkeitsfragen bei neutronenstreumessungen, Nukleonik 8 (1966) 61.

[3] A. Meyer, R. M. Dimeo, P. M. Gehring, D. A. Neumann, The high-flux backscattering spectrometer at the NIST center for neutron research, Rev. Sci. Instrum. 74 (2003) 2759-2777. doi:10.1063/1.1568557.

[4] O. Kirstein, T. Kozielewski, M. Prager, D. Richter, Status of the highflux backscattering spectrometer RSSM for the FRM-II reactor in munich, Appl. Phys. A 74 (2002) S133-S135. doi:10.1007/s003390201536.

[5] J. Wuttke, A. Budwig, M. Drochner, H. Kämmerling, F.-J. Kayser, H. Kleines, V. Ossovyi, L. C. Pardo, M. Prager, D. Richter, G. J. Schneider, H. Schneider, S. Staringer, SPHERES, jülich's high-flux neutron backscattering spectrometer at FRM II, Rev. Sci. Instrum. 83 (2012) 075109. doi:10.1063/1.4732806.

[6] M. Hennig, B. Frick, T. Seydel, Optimum velocity of a phase-space transformer for cold-neutron backscattering spectroscopy, J. Appl. Cryst. 44 (2011) 467-472. doi:10.1107/S0021889811013227.

[7] C. J. Carlile, M. A. Adams, The design of the IRIS inelastic neutron spectrometer and improvements to its analysers, Physica B: Condens. Matter 182 (1992) 431-440. doi:10.1016/0921-4526(92)90047-V.

[8] C. J. Carlile, M. A. Adams, P. S. R. Krishna, M. Prager, K. Shibata, P. Westerhuijs, Less background, better contrast by cooling analyser crystals, Nucl. Instr. and Meth. in Phys. Res. A 338 (1994) 78-82. doi:10.1016/0168-9002(94)90165-1.

[9] D. Martín y Marero, S. Campbell, C. J. Carlile, The OSIRIS polarisation analysis spectromter and diffractometer, J. Phys. Soc. Jpn. 65 (1996) $245-248$.

[10] M. T. F. Telling, K. H. Andersen, Spectroscopic characteristics of the OSIRIS near-backscattering crystal analyser spectrometer on the ISIS pulsed neutron source, Phys. Chem. Chem. Phys. 7 (2005) 1255-1261. doi:10.1039/B413934H. 
[11] E. Mamontov, K. W. Herwig, A time-of-flight backscattering spectrometer at the spallation neutron source, BASIS, Rev. Sci. Instrum. 82 (2011) 085109. doi:10.1063/1.3626214.

[12] K. Shibata, N. Takahashi, K. Kawakita, M. Matsuura, T. Yamada, T. Tominaga, W. Kambara, M. Kobayashi, Y. Inamura, T. Nakatani, K. Nakajima, M. Arai, The performance of TOF near backscattering spectrometer DNA in MLF, J-PARC, Jap Phys Soc Conf Proc 8 (2015) 036022. doi:doi.org/10.7566/JPSCP.8.036022.

[13] N. Takahashi, K. Shibata, T. J. Sato, Y. Kawakita, I. Tsukushi, N. Metoki, K. Nakajima, M. Arai, Study of the analyzer crystals for use in the near-backscattering spectrometer DNA at JPARC, Nucl. Instrum. Methods Phys. Res. A 600 (2009) 91-93. doi:10.1016/j.nima.2008.11.057.

[14] M. Arai, R. Kajimoto, M. Nakamura, Y. Inamura, K. Nakajima, K. Shibata, N. Takahashi, J. Suzuki, S. Takata, T. Yamada, S. Itoh, Recent developments of instruments in a spallation neutron source at J-PARC and those prospects in the future, J. Phys. Soc. Jpn. 82 (2013) SA024. doi:10.7566/JPSJS.82SA.SA024.

[15] N. Tsapatsaris, R. E. Lechner, M. Markó, H. N. Bordallo, Conceptual design of the time-of-flight backscattering spectrometer, MIRACLES, at the european spallation source, Rev. Sci. Instrum. 87 (2016) 085118. doi:10.1063/1.4961569.

[16] F. Demmel, K. H. Andersen, FIRES: a novel neutron backscattering spectrometer, Meas. Sci. Technol. 19 (2008) 034021. doi:10.1088/09570233/19/3/034021.

[17] F. Demmel, P. Bernhardt, A. Magerl, E. Steichele, A high resolution TOF diffractometer and spectrometer, Physica B 276-278 (2000) 116117. doi:10.1016/S0921-4526(99)01266-1.

[18] L. van Eijck, L. Gérard, B. Frick, T. Seydel, H. Schober, A case study for using neutron backscattering instruments at reactors in inverted timeof-flight mode, Nucl. Instrum. Methods Phys. Res. A 672 (2012) 64-68. doi:10.1016/j.nima.2011.12.081. 
[19] P. L. W. Tregenna-Piggott, F. Juranyi, P. Allenspach, Introducing the time-of-flight backscattering instrument MARS at SINQ, Neutron News 19 (2008) 20-24. doi:10.1080/10448630701832001.

[20] M. Appel, B. Frick, A. Magerl, A flexible high speed pulse chopper system for an inverted neutron time-of-flight option on backscattering spectrometers, Sci. Rep. 8 (2018) 13580. doi:10.1038/s41598-018-31774y.

[21] D. Martín y Marero, Towards high resolution polarisation analysis using double polarisation and ellipsoidal analysers, Appl. Phys. A 74 (2002) s289-s291. doi:10.1007/s003390201802.

[22] K. H. Andersen, D. M. y Marero, M. J. Barlow, The OSIRIS diffractometer and polarisation-analysis backscattering spectrometer, Appl. Phys. A Mater. Sci. Process. 74 (2002) S237-S239. doi:10.1007/s003390201804.

[23] F. Demmel, D. McPhail, J. Crawford, D. Maxwell, K. Pokhilchuk, V. Garcia-Sakai, S. Mukhopadyay, M. T. F. Telling, F. J. Bermejo, N. T. Skipper, F. Fernandez-Alonso, Opening the terahertz window on the OSIRIS spectrometer, EPJ Web of Conferences 83 (2015) 03003. doi:10.1051/epjconf/20158303003.

[24] F. Demmel, K. Pokhilchuk, The resolution of the tof-backscattering spectrometer OSIRIS: monte carlo simulations and analytical calculations, Nucl. Instr. Meth. A 767 (2014) 426-432. doi:10.1016/j.nima.2014.09.019.

[25] C. Stock, C. Broholm, Y. Zhao, F. Demmel, H. J. Kang, K. C. Rule, C. Petrovic, Magnetic field splitting of the spin resonance in $\mathrm{CeCoIn}_{5}$, Phys. Rev. Lett. 109 (2012) 167207. doi:10.1103/PhysRevLett.109.167207.

[26] W. J. Gannon, I. A. Zaliznyak, L. S. Wu, A. E. Feiguin, A. M. Tsvelik, F. Demmel, Y. Qiu, J. R. D. Copley, M. S. Kim, M. C. Aronson, Spinon confinement and a sharp longitudinal mode in $\mathrm{Yb}_{2} \mathrm{Pt}_{2} \mathrm{~Pb}$ in magnetic fields, Nat. Comm. 10 (2019) 1123. doi:10.1038/s41467-019-08715-y.

[27] A. J. O'Malley, M. Sarwar, J. Armstrong, C. R. A. Catlow, I. P. Silverwood, A. P. E. York, I. Hitchcock, Comparing ammonia diffusion in 
NH3-SCR zeolite catalysts: a quasielastic neutron scattering and molecular dynamics simulation study, Phys. Chem. Chem. Phys. 20 (2018) 11976-11986. doi:10.1039/C8CP01022F.

[28] I. P. Silverwood, S. F. Parker, C. R. A. Catlow, Neutron scattering in catalysis and energy materials, Phys. Chem. Chem. Phys. 18 (2016) 17140-17140. doi:10.1039/C6CP90150F.

[29] T. J. Willis, D. G. Porter, D. J. Voneshen, S. Uthayakumar, F. Demmel, M. J. Gutmann, M. Roger, K. Refson, J. P. Goff, Diffusion mechanism in the sodium-ion battery material sodium cobaltate, Scientific Reports 8 (2018) 3210. doi:10.1038/s41598-018-21354-5.

[30] F. Demmel, D. McPhail, C. French, D. Maxwell, S. Harrison, J. Boxall, N. Rhodes, S. Mukhopadhyay, I. Silverwood, V. García Sakai, F. Fernandez-Alonso, TOF-backscattering spectroscopy at the ISIS facility: Status and perspectives, J. of Phys. Conf. Series 1021 (2018) 012027. doi:10.1088/1742-6596/1021/1/012027.

[31] B. Frick, E. Mamontov, L. van Eijck, T. Seydel, Recent backscattering instrument developments at the ILL and SNS, Zeit. Phys. Chem. 224 (2010) 33-60. doi:10.1524/zpch.2010.6091.

[32] R. H. Scherm, E. Krüger, Bragg optics - focusing in real and $k$-space, Nucl. Instr. and Meth. in Phys. Res. A 338 (1994) 1-8. doi:10.1016/01689002(94)90154-6.

[33] J. Kalus, B. Dorner, On the use of in-pile collimators in inelastic neutron scattering, Acta Cryst. A 29 (1973) 526-528. doi:10.1107/S0567739473001312.

[34] P. Willendrup, E. Farhi, E. Knudsen, U. Filges, K. Lefmann, McStas: Past, present and future, J. Neutron Res. 17 (2014) 35-43. doi:10.3233/JNR-130004.

[35] P. Willendrup, E. Farhi, K. Lefmann, McStas 1.7 - a new version of the flexible monte carlo neutron scattering package, Physica B 350 (2004) E735-E737. doi:10.1016/j.physb.2004.03.193. 
[36] K. Lefmann, K. Nielsen, McStas, a general software package for neutron ray-tracing simulations, Neutron News 10 (1999) 20-23. doi:10.1080/10448639908233684.

[37] A. Lovell, F. Fernandez-Alonso, N. T. Skipper, K. Refson, S. M. Bennington, S. F. Parker, Quantum delocalization of molecular hydrogen in alkali-graphite intercalates, Phys. Rev. Lett. 101 (2008) 126101. doi:10.1103/PhysRevLett.101.126101.

[38] F. Fernandez-Alonso, F. J. Bermejo, C. Cabrillo, R. O. Loutfy, V. Leon, M. L. Saboungi, Nature of the bound states of molecular hydrogen in carbon nanohorns, Phys. Rev. Lett. 98 (2007) 215503. doi:10.1103/PhysRevLett.98.215503. 

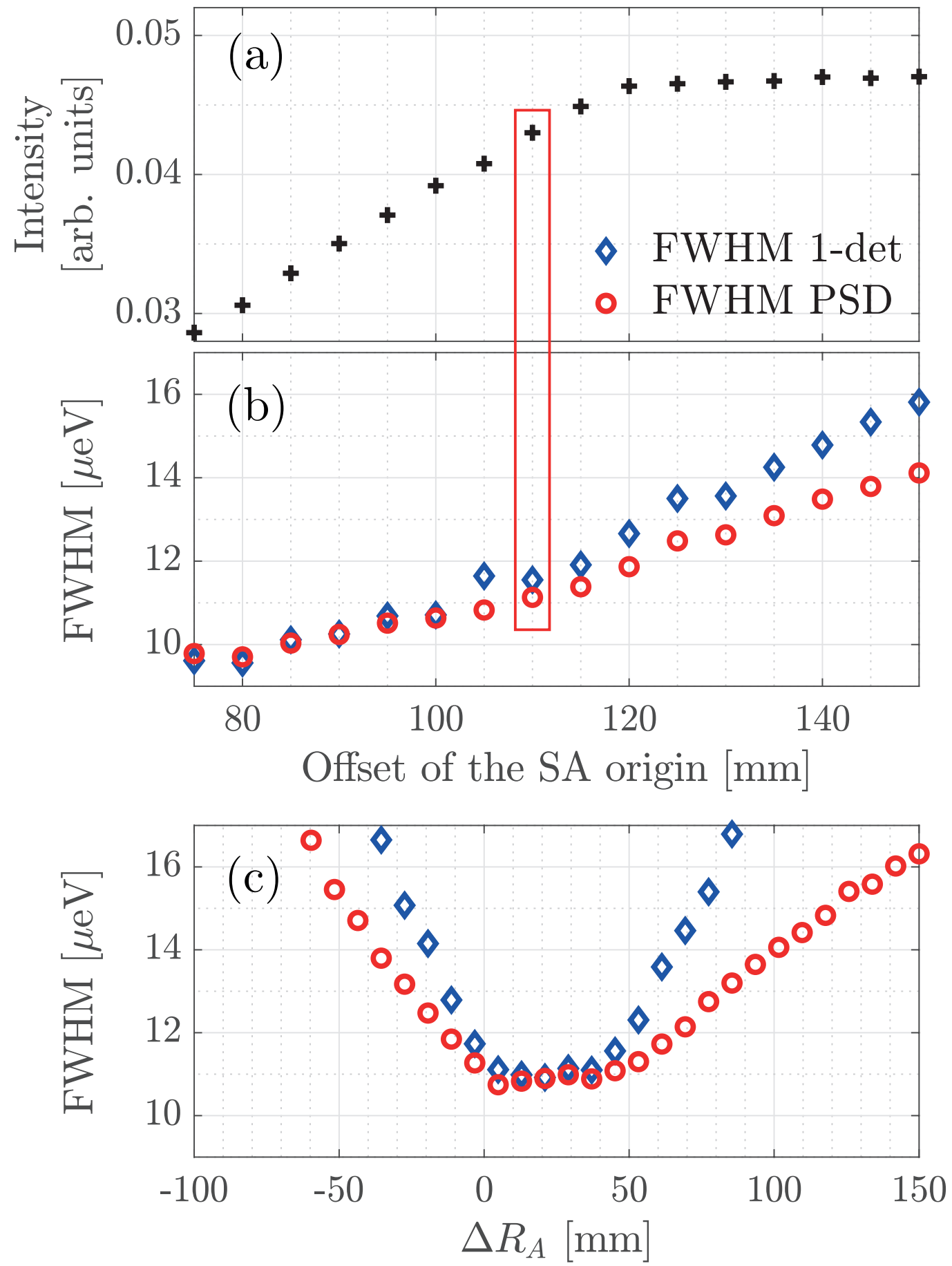

Figure 5: Dependence of the intensity (a) and FWHM (b) of the line shape with the offset of the SA origin. Included are simulated results from a whole detector tube (diamonds) and from the summed individual pixels (cirgles). (c) Dependence of the FWHM with the $\Delta R_{A}$ parameter. 


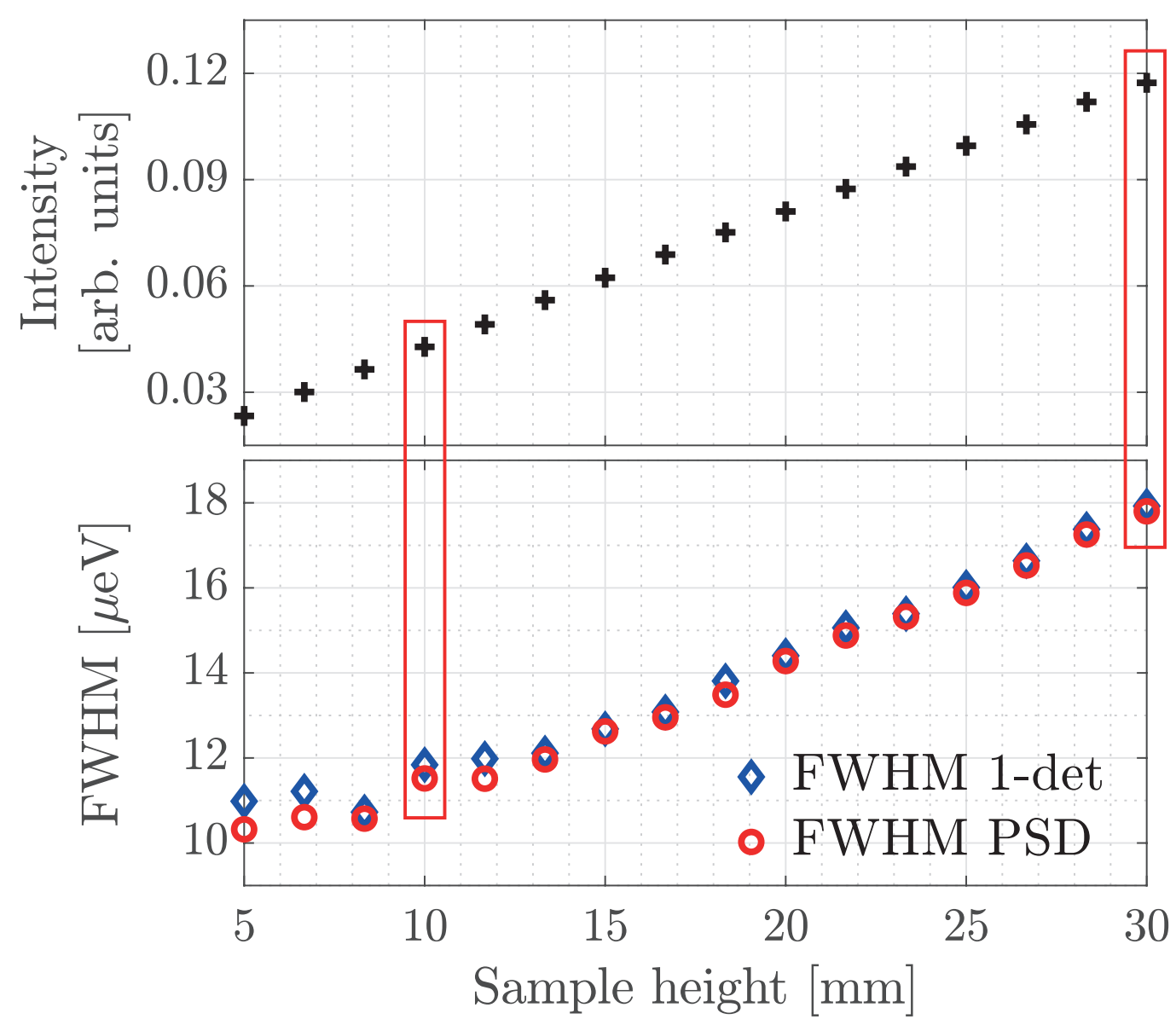

Figure 6: Dependence of the intensity and FWHM on sample height. Included are simulated results from a whole detector tube (diamonds) and from the summed individual pixels (circles). 


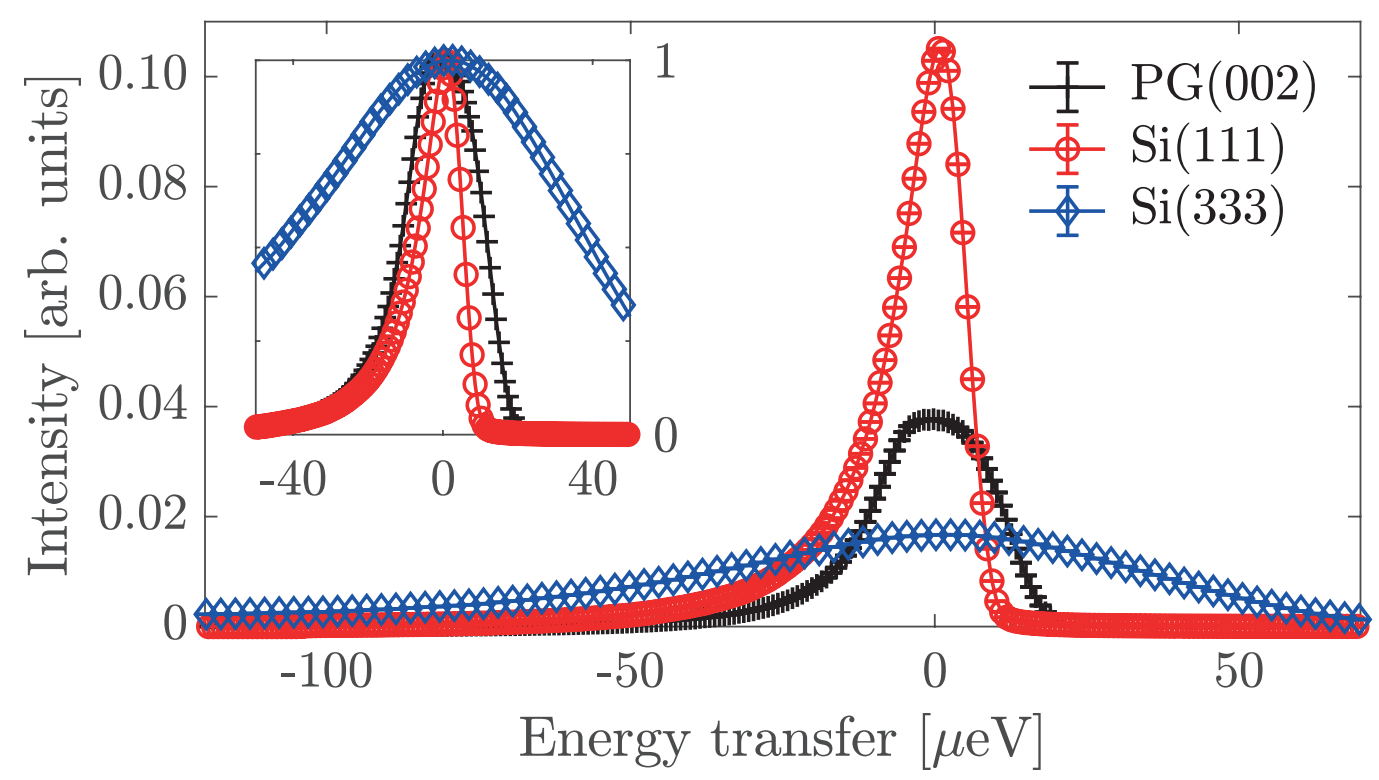

Figure 7: Line shape for the PG(002) (crosses), and average line shapes for the SA (top and bottom), considering the (111) reflection (circles) and the (333) reflection (diamonds). Line shapes are normalised to unity in insert. 